\title{
Molecular Topology QSAR Strategy for Crop Protection: New Natural Fungicides with Chitin Inhibitory Activity
}

\author{
Maria Galvez-Llompart, Riccardo Zanni, Jorge Galvez, and Ramon Garcia-Domenech*
}

Cite This: ACS Omega 2020, 5, 16358-16365

Read Online

ABSTRACT: Nowadays, crop protection is a major concern and how to proceed is a delicate point of contention. New products must be safe and ecofriendly in accordance with the actual legislation. In this context, we present a quantitative structure-activity relationship strategy based on molecular topology as a tool for generating natural products as potential fungicides following a mechanism of action based on the synthesis of chitin inhibition (chitinase inhibition). Two discriminant equations using statistical linear discriminant analysis were used to identify three potential candidates (1-methylxanthine, hematommic acid, and antheraxanthin). The equations showed accuracy and specificity levels above $80 \%$, minimizing the risk of selecting false active compounds.

\section{INTRODUCTION}

Nowadays fungi cause crop losses worldwide, with fungal diseases having a significant economic impact on plant yield and quality; thus, managing such diseases is an essential component of production for most crops. ${ }^{1,2}$ The identification of molecules targeting enzymes and/or biochemical processes almost exclusive in fungi is a useful approach for agrochemical companies when fighting resistant infestations. Fungal cell walls are primarily composed of polysaccharides, namely chitin, glucans, mannans, and glycoproteins. Chitin is one of the main components, and it is not present in plant cells. ${ }^{3}$ Basically, it is a eukaryotic extracellular amino sugar biopolymer, massively produced by most fungal systems and by invertebrates, notably arthropods. For its importance in fungi life cycle, chitin has been considered as a selective target for pesticide action. ${ }^{4}$ It is synthesized by chitin synthase and cleaved by chitinase which degrades or remodels the cell wall. This way, it is capable to maintain cell wall plasticity during growth. Fungal chitinases are crucial for cell division and replication and also for cell wall remodeling. In addition, they play an important role in cell nutrition, morphogenesis, and development processes; so, the importance as potential targets is clear. ${ }^{5,6}$ Chitin synthesis inhibitors were considered new since $1977 .^{7}$ Over the past few decades, a variety of chitinase inhibitors were identified and characterized. The majority of potent chitinase inhibitors are naturally derived products such as pseudotrisaccharide allosamidin and cyclic peptides such as argifin, psammaplin, and argadin. Chemically, many reported chitinase inhibitors share the imine functionality, either in acyclic or cyclic form. ${ }^{6}$ Moreover, several diverse mechanisms of action (MOA) related to chitin synthase have been described till today:

- ompetition of the analogues of UDP- $N$-acetylglucosamine with the substrate.
- Prevention of the permeation of the substrate through the cytoplasmic membrane so that it is unable to reach the target enzyme.

- Inhibition of chitin biosynthesis and chitin deposition.

- Postpolymerization event, the translocation of chitin chains across the cell membrane.

- Stimulation of the immune system of the plant by inhibiting chitin-related enzymes, the plant elicitors.

The last one is probably the latest and one of the most interesting MOA. The present paper presents two quantitative structure-activity relationship (QSAR) discriminant equations, designed by molecular topology (MT), as tools to identify the potential fungicides and new inhibitors of chitin formation. The MT paradigm consists in characterizing molecules through numerical descriptors called topological indices. These descriptors are based on simple and intuitive notions of a structure having to do with connections between different atoms in a molecule (molecular connectivity). The key elements are not geometrical or physical features of molecules, such as interatomic distances, bond angles or energies, and so forth (which usually play a main role in most QSAR approaches), but features such as the number of links between two atoms, the presence or absence of cycles, the possibility of connecting two particular atoms by a certain path, and so forth. ${ }^{8,9}$ It can be said that MT goes beyond standard QSAR approaches, allowing the generation of a huge number

Received: January 14, 2020

Accepted: March 23, 2020

Published: June 26, 2020 
of descriptors and increasing the chance of strong correlations. In addition, it must be noted that topological descriptors only rely on structure; so, no experimental measures are required. They are rentable, flexible (it is possible to use topological descriptors for every kind of structure), and contrary to the general assumption, and they can be used to predict threedimensional properties. ${ }^{10}$ Latest results showed the potential of MT in antimicrobial resistance control, ${ }^{11}$ fungi resistance control, $^{12}$ and biodegradability prediction ${ }^{13}$ as a powerful tool for research related with health, crop, and environment safety.

\section{MATERIALS AND METHODS}

The MT approach consists of the following steps

- Step I: creation of a specific database. Compounds with fungicide and chitin synthase inhibition activity were retrieved from the literature and "The pesticide manual" reported by the British Crop Protection Council. ${ }^{14}$

- Step II: calculation of topological descriptors using the Dragon software. ${ }^{15}$

- Step III: division of the data set into two groups: training and test sets.

- Step IV: calculation of the topological predictive models, applying the linear discriminant analysis (LDA) technique: $\mathrm{DF}_{1}$ and $\mathrm{DF}_{2}{ }^{16}$

- Step V: performance of external and internal validation to assess the robustness of the predictive equations.

- Step VI: virtual screening of database (The Natural Product Collection from MicroSource Discovery System Inc. $)^{17}$

- Step VII: selection of potential candidates as fungicides with inhibitory activity against the chitin formation.

Compound Analysis. The first QSAR model $\left(\mathrm{DF}_{1}\right)$ was developed from a database of 295 heterogeneous active and inactive structural compounds with fungicidal activity. The data were split into two subsets: training set (215 molecules) and external test set ( 80 molecules). Each set was again split into two groups: active and inactive, taking into account different chemical features in order to reach a coherent balance on chemical diversity between the groups. All active compounds were collected from the literature. ${ }^{14}$ The second QSAR model $\left(\mathrm{DF}_{2}\right)$ was calculated using a dataset of 41 molecules (training set) retrieved from the literature. ${ }^{18,19}$ For both models, the inactive molecules were retrieved from the Sigma-Aldrich compound collection. After a comprehensive analysis of the dataset, a study of molecular descriptors is provided.

Molecular Descriptors. Topological descriptors codify information about the molecular structure in a purely numerical way. The numerical format makes the search of new hits and leads easier. ${ }^{20}$ The structure of molecules was drawn using ChemDraw Ultra (version 10.0) ${ }^{21}$ and characterized by a set of descriptors such as constitutional and topological descriptors. Among the last stand edge-adjacency indices, walk and path counts, connectivity indices, and topological charge indices. ${ }^{22}$ Other graph-theoretical descriptors were also calculated but not depicted here because of their lack of effectiveness. All indices were calculated with the Dragon software version 5.4, ${ }^{15}$ and their values for the selected equations for every compound included in this study (training set, external test set, and virtual screening set) are shown in the Supporting Information.
Modeling Techniques. In the present work, LDA was used to calculate QSAR models. LDA allows calculation of a discriminant function (DF), which best separates two categories or objects in a specific case into active and inactive groups. ${ }^{23}$ The software used was Statistica 9.0. ${ }^{16}$ Discriminant capability was assessed as the percentage of correct classifications in each set of compounds. Its classification criterion is based on the minimum Mahalanobis distance (the distance of each case to the mean of all cases in a category), ${ }^{24}$ and the quality of discrimination was evaluated using Wilks' lambda $(\lambda)$ parameter, ${ }^{25}$ which is related with the multivariate analysis of variance that tests the equality of group means for the variable in the discriminant model. The smaller the Wilks parameter value, the smaller is the overlap between the active and inactive groups $(\lambda=0$ would mean a perfect separation between the groups). The descriptor selection was carried out according to the Fisher-Snedecor parameter $(F){ }^{26}$ which establishes the relevance of candidate variables. The stepwise procedure is "guided" by the respective $F$ to introduce and remove values. The $F$ value for a variable indicates its statistical significance in the discrimination between groups, that is, it is a measure of the extent to which a variable makes a unique contribution to the prediction of group membership. The descriptors included to compute the linear classification function are chosen in a stepwise manner: at each step, the variable making the largest contribution in discriminating between groups is introduced into the equation (or the variable that makes the smallest contribution is removed).

Model Validation. Validation of DFs was performed using external (test set) and internal ( $y$-randomization) validation techniques. In addition, the sensitivity, specificity, and predictive precision of the models are reported.

In order to determine the capability of the model for identifying true positive compounds (true active), sensitivity has been calculated, following the formula: sensitivity = $($ true $P /($ true $P+$ FN $)) \times 100$. Furthermore, to assess the capability of the model in identifying true negative (true inactive) compounds, the specificity has also been calculated: specificity $=($ trueN $/($ trueN + FP $)) \times 100$. Finally, the model accuracy is calculated to quantify the ability to identify the true positives and negatives (real active and inactive compounds): accuracy $=((\operatorname{true} P+\operatorname{trueN}) /(\operatorname{true} P+\mathrm{FN})+(\operatorname{trueN}+\mathrm{FP})) \times$ 100.

ROC Curve. Receiver operating characteristic (ROC) curve is a graphical display of sensitivity on $y$-axis and (1specificity) on $x$-axis for varying cutoff points of values. ${ }^{27}$ It is depicted as a square box, with both axes from 0 to 1 . The area under the curve (AUC) is a measure of sensitivity and specificity for assessing the robustness of a predictive test. Maximum AUC $=1$ means the prediction test is perfect in differentiating active cases from inactive cases. This implies that both sensitivity and specificity are 1 and that both errors-false positive and false negative-are 0 . The closer AUC is to 1 , the better is the performance of the test. The diagonal joining the point $(0,0)$ to $(1,1)$ divides the square in two equal parts, and each has an area equal to 0,5 . When the ROC meets with this line ( $A U C=0,5)$, there is a $50-50$ chance that the test will discriminate the active and nonactive cases: random classification. ${ }^{27}$

Activity Distribution Diagram. LDA in topological QSAR allows plotting frequency distribution diagrams. The diagrams represent a function of the number of molecules within an interval of values of DF versus these values. It is 
called activity distribution diagram (ADD). For structurally heterogeneous groups of molecules, the diagram shows skewed Gaussian shapes or present several maxima. The maxima afford intervals of DF in which exists a good expectancy to find new active molecules. $^{28}$

Virtual Screening. Once a maximum expectancy range of DF for finding fungicide and chitin inhibitor activities is defined, a virtual screening of a commercial database is performed. The natural product collection from MicroSource Discovery System Inc. ${ }^{17}$ was used here to find new natural compounds with fungicide activity and inhibitory activity of chitin formation.

\section{RESULTS AND DISCUSSION}

QSAR Topological Models. The two QSAR models, one for predicting fungicidal activity $\left(\mathrm{DF}_{1}\right)$ and the other for predicting the inhibitory activity of chitin formation $\left(\mathrm{DF}_{2}\right)$, are presented below. The first $\mathrm{DF}\left(\mathrm{DF}_{1}\right)$, a six-variable equation, is reported

$$
\begin{aligned}
\mathbf{D F}_{1}= & (\mathbf{D} / \text { Dr05 } \times 0.020)+(\text { piPC04 } \times 1.319) \\
& -(\text { MPC10 } \times 0.037)-(\text { EEig03r } \times 2.862) \\
& +(\text { EEig14r } \times 2.366)+(\text { JGI3 } \times 23.364)+3.321
\end{aligned}
$$

The parameters accounting for the significance of this equation were

$$
N=215 ; \lambda=0.732 ; \quad F=12.7 ; p<0.00001
$$

where $N$ is the number of training set compounds; $\lambda$, Wilks' lambda; F, Fisher-Snedecor parameter; $p$, statistical significance; $D / D r 05$, distance/detour ring index of order 5; piPC04, molecular multiple path count of order 4; MPC10, molecular path count of order 10; EEig3r, eigenvalue 3 from edge adjacency matrix weighted by resonance integrals; EEig14r, eigenvalue 14 from edge adjacency matrix weighted by resonance integrals; and JGI3, the mean topological charge index of order 3.

Although it is not easy to establish an explanation in terms of structure-activity relationship for an equation with as many variables as $\mathrm{DF}_{1}$, some observations can be made. Among the descriptors, D/Dr05, pIPC04, EEig14r, and JGI3 contribute positively to fungicidal activity, wheeas MPC10 and EEig3r contribute negatively. $\mathrm{D} / \mathrm{Dr} 05$, for example, the distance/ detour ring index of order 5 is a topological descriptor. The positive coefficient of this descriptor suggests that the presence of five-member rings contribute to increase the fungicidal activity. Although the presence of five-member rings may not be a conditio sine qua non for fungicidal activity, it can be observed how compounds that do have five-member rings and values of $D / \operatorname{Dr} 05(45-150)$ are classified as active by the model. On the contrary, compounds with values of the descriptor $<45$ or $>150$ are classified as inactive (Figure 1).

piPC04, a member of the walk and path family descriptors, is defined as the sum of weights of the paths of length 4 . Therefore, the piPC04 value is expected to increase according to the increasing size, branching, and length of carbon chains in molecules (Figure 2). When analyzing the value adopted by this index in the training set, it can be seen how molecules with values of piPC04 (0-2.8) are always classified as fungicides by the model. However, compounds which adopt values (2.85.4) are classified as active or inactive depending on the overall

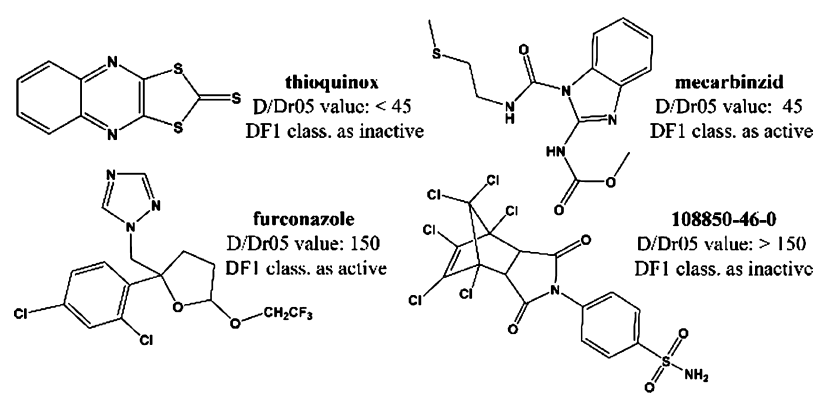

Figure 1. Analysis of D/Dr05 values for training set compounds.

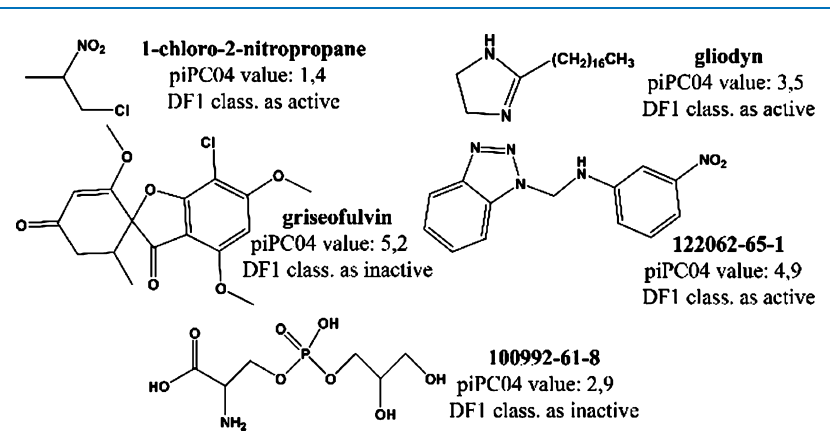

Figure 2. Analysis of piPC04 values for training set compounds.

calculation of the rest of the descriptors present in the equation (Figure 2).

JGI3 (mean topological charge index of order 3) is a descriptor that provides information about the exchange of charges between atoms located at a topological distance 3, per bond, that is, it represents an average value of the charge transferred at distance 3 in the molecule.

In addition, it should be noted how the descriptors that less contribute to the discrimination of fungicidal activity are two of the three most statistically relevant in the model $(F>25)$. MPC10 provides pure topological information of the compounds, whereas EEig3r is related with the resonant effect. Finally, the descriptor which contributes greater to the model is EEig14r $(F>30)$, which describes the degree of conjugation of the molecules related with the resonance effect. In fact, as shown in Figure 3, molecules with higher conjugation (Blasticidin-S or S102385) adopt larger values of these indices than less-conjugated ones (ampropylfos or 1067-47-6).

All the compounds of the training and test sets are reported in Table S1 in the Supporting Information, showing: the

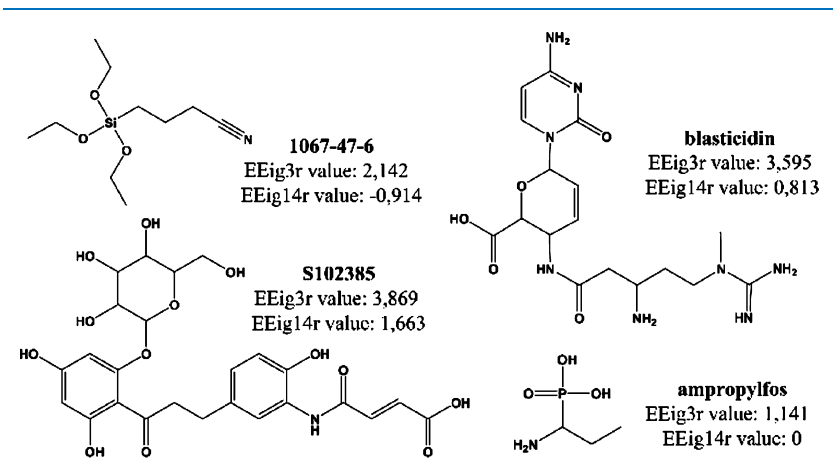

Figure 3. Analysis of the resonance effect by EEig3r and EEig14r indexes. 
probability of activity, the $\mathrm{DF}_{1}$ value, and the value of each respective descriptor of the discriminant equation.

According to the results of $\mathrm{DF}_{1}$, a molecule will be classified as a potential fungicide if $\mathrm{DF}>0$ and as a nonfungicide if $\mathrm{DF}<$ 0 . By applying this criterion to the training set of 215 compounds, 73 out of 104 active compounds were correctly classified as fungicides ( $70.2 \%$ accuracy), whereas 90 out of 111 inactive compounds were correctly classified $(81.1 \%$ accuracy), as can be seen in Table 1 . To evaluate the

Table 1. Classification Matrix Obtained through the Selected $\mathrm{DF}_{1}$ and $\mathrm{DF}_{2}$ for the Training and External Test Set

\begin{tabular}{lccc} 
& \% correct class* & no active class & no inactive class \\
& \multicolumn{1}{c}{ Training Set $\left(\mathrm{DF}_{1}\right)$} & \\
active group & 70.2 & 73 & 31 \\
inactive group & 81.1 & 21 & 90 \\
& External Test Set $\left(\mathrm{DF}_{1}\right)$ & \\
active group & 67.5 & 27 & 13 \\
inactive group & 77.5 & 9 & 31 \\
& Training Set $\left(\mathrm{DF}_{2}\right)$ & \\
active group & 82.3 & 14 & 3 \\
inactive group & 83.3 & 4 & 20 \\
\hline
\end{tabular}

robustness of the discriminant equation, an external validation was run using an external test set. The group was made up of 80 compounds (40 active and 40 inactive, as fungicides, respectivel/y) which were not included in the training set. A random selection of about $30 \%$ of all dataset was made. The model yields a correct classification of $67.5 \%$ for the active group (27 out of 40 compounds) and $77.5 \%$ for the inactive set ( 31 out of 40 compounds), as shown in Table 1 .

As illustrated in Table 1 , the model has a higher specificity than sensitivity both for the training and test sets (81.1 and $77.5 \%$, respectively). This is important because it minimizes the risk of selecting false active compounds. Figure 4 gives visual insights about the whole specificity and sensitivity of the model.

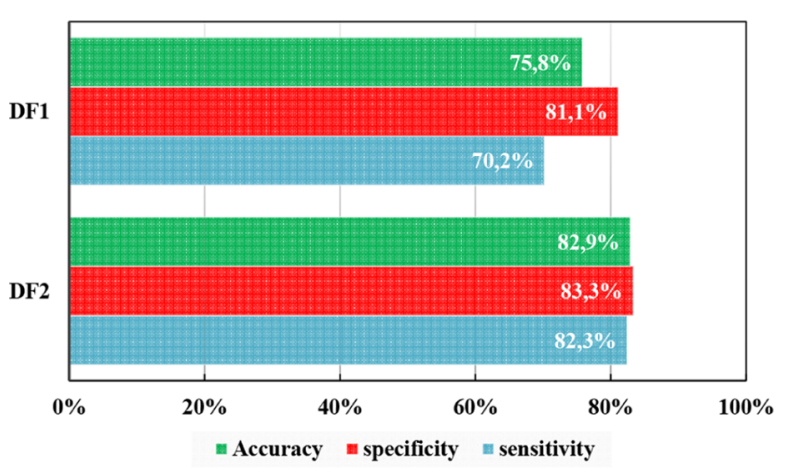

Figure 4. Graphic of the accuracy, specificity, and sensitivity for $\mathrm{DF}_{1}$ and $\mathrm{DF}_{2}$.

From these results, it is clear that when using the model to screen the database for potential antifungal agents, there is an $18.9 \%$ probability of selecting false active compounds. Figure $5 \mathrm{~A}$ shows the ROC curve for $\mathrm{DF}_{1}$. A value of $\mathrm{AUC}=0.893$ represents an $89.3 \%$ probability that two compounds, one active and the other inactive, are correctly classified by the $\mathrm{DF}_{1}$ function.
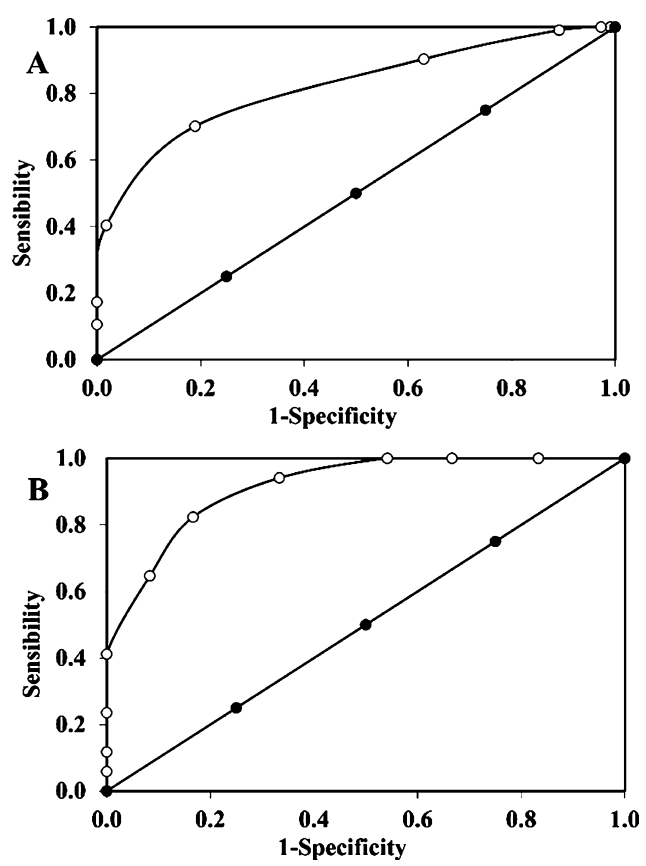

Figure 5. ROC curves for $\mathrm{DF}_{1}(\mathrm{~A})$ and $\mathrm{DF}_{2}$ (B) for different thresholds of class function (between -10 and +10$)$. AUC $\left(D_{1}\right)=$ 0.893 and AUC $\left(\mathrm{DF}_{2}\right)=0.946$.

To identify the $\mathrm{DF}_{1}$ applicability domain, $\mathrm{ADD}$ is developed. $^{28}$ Molecules showing fungicide activity are represented in black, whereas inactive molecules are represented in white. In Figure $6 \mathrm{~A}$ it can be seen how the model's range of applicability is for $\mathrm{DF}_{1}$ values between -6 and 4. In addition, the graphic gives information about the range of $\mathrm{DF}_{1}$ in which the expectancy of finding fungicides is higher $(0.5-4.0)$. Furthermore, a zone of overlap between the
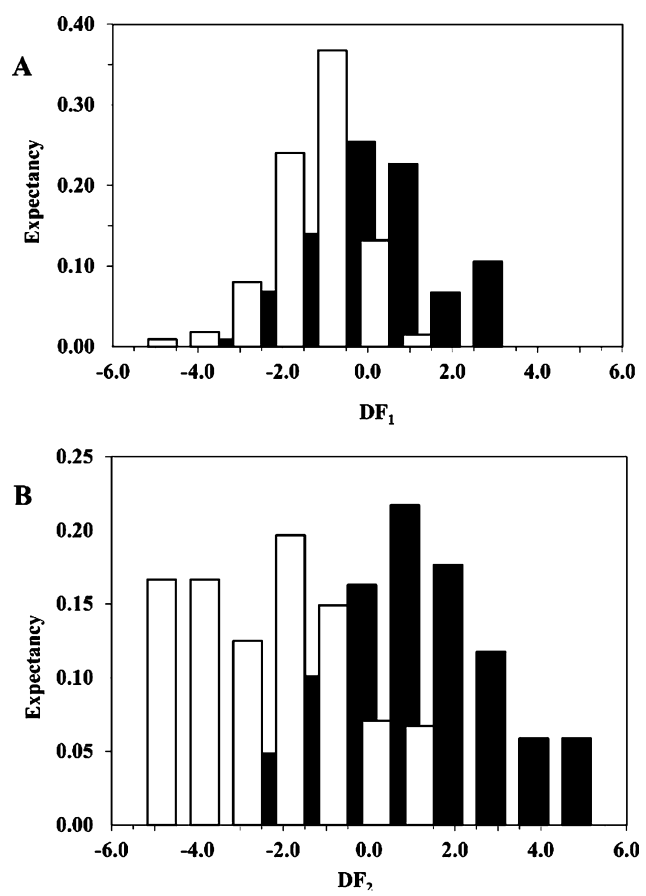

Figure 6. $\mathrm{ADD}$ for $\mathrm{DF}_{1}(\mathrm{~A})$ and $\mathrm{DF}_{2}(\mathrm{~B})$ (the black color represents the fungicide or inhibitor of the chitin formation compounds, and the white color compounds without these activities). 
fungicidal and nonfungicidal compounds $(0.5$ and -3.5$)$ is present. $\mathrm{DF}_{1}$ values $<-6$ and $>4.0$ will be considered outside the range of applicability of the model; therefore, these molecules are nonclassifiable by the model.

Once the fungicidal activity is modeled, the other main objective of the present work is addressed: to develop a predictive model to find the potential inhibitors of chitin formation. A new discriminant equation of three descriptors was made (eq 2)

$$
\begin{aligned}
\mathrm{DF}_{2}= & (\text { ATS4m } \times 13.534)-(\text { ATS5m } \times 8.907) \\
& -(\text { GATS5m } \times 3.040)-17.050
\end{aligned}
$$

The statistics of this equation were

$$
N=41 ; \lambda=522 ; F=11.3 ; p<0.00001
$$

where $N$ is the number of training set compounds; $\lambda$, Wilks' lambda; F, Fisher-Snedecor parameter; $p$, statistical significance; ATS4m, the Broto-Moreau autocorrelation index of topological structure - lag 4/weighted by atomic masses; ATS5m, the Broto-Moreau autocorrelation index of topological structure - lag 5/weighted by atomic masses; and GATS5m, the Geary autocorrelation index - lag 5/weighted by atomic masses.

The descriptors in $\mathrm{DF}_{2}$ are related to the atomic mass of atoms present in the molecules. As illustrated in Figure 7,

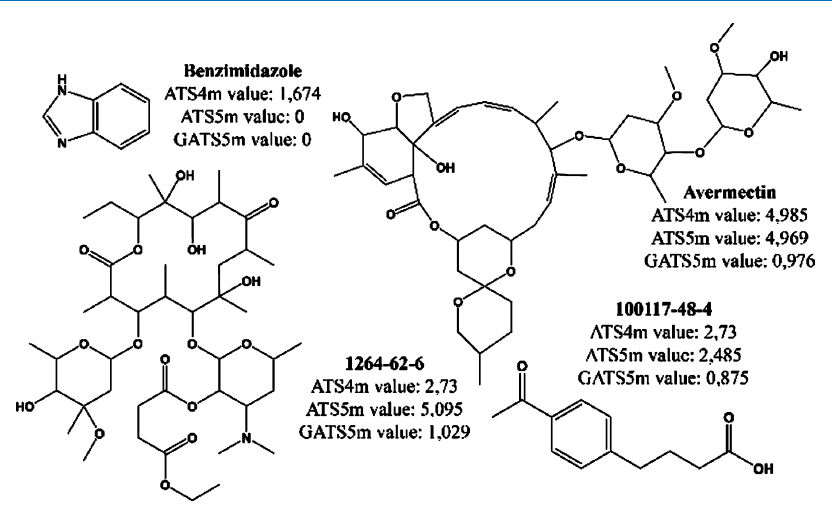

Figure 7. Analysis of index related to atomic mass in $\mathrm{DF}_{2}$.

molecules with a larger size, and therefore larger atomic mass, adopt higher values of the descriptors in the model for predicting the inhibitory activity against chitin. However, there is no direct relationship between a greater value of these descriptors and a greater value of $\mathrm{DF}_{2}$ because two of the descriptors (ATS5m and GATS5m) contribute negatively to the equation.

All the compounds of the training set are reported in Table S2 in the Supporting Information, showing the probability of activity, the $\mathrm{DF}_{2}$ value, and the value of each respective descriptor in the discriminant equation.

The discriminant model classifies those molecules with $\mathrm{DF}_{2}$ $>0$ as potential inhibitors of chitin formation, whereas those with $\mathrm{DF}_{2}<0$ as inactive. By applying this criterion to the training set (41 compounds), 14 out of 17 have been correctly classified as inhibitors of chitin formation ( $82.3 \%$ accuracy), and 20 out of 24 inactive compounds were also correctly classified ( $83.3 \%$ accuracy), as can be seen in Table 1 .

As shown in Table 1 and Figure $4, \mathrm{DF}_{2}$ shows high specificity and sensitivity. $\mathrm{DF}_{2}$ has a probability to select false active and inactive compounds for values of DF $>0$ or $<0$ of $17 \%$.

Considering the small size of the database of chitin formation inhibitors, the external validation of the model could not be done. Therefore, an internal validation was performed. $y$-Scrambling or $y$-randomization test was performed, in which 10 molecules between the active and inactive groups are interchanged randomly. This is a form of arrangement test, where the values of the dependent variable (y) are randomly assigned to different compounds, whereas the descriptor values ( $x$ 's) are left unchanged. ${ }^{29}$ The rearranged data are then used for training QSAR models. As shown in Table 2 , the value of $\lambda$ (Wilks) increases significantly in all the

Table 2. $y$-Randomization Test Performed to $\mathrm{DF}_{2}$

$\begin{array}{lrcc}\text { series } & \lambda(\text { Wilks }) & \text { sensitivity }(\%) & \text { specificity }(\%) \\ \text { Training } & \mathbf{0 . 5 2 2} & \mathbf{8 2 . 3} & \mathbf{8 3 . 3} \\ \text { random_1 } & 0.816 & 64.7 & 66.7 \\ \text { random_2 } & 0.939 & 64.7 & 62.5 \\ \text { random_3 } & 0.812 & 58.8 & 66.7 \\ \text { random_4 } & 0.828 & 64.7 & 70.8 \\ \text { random_5 } & 0.837 & 77.8 & 69.6\end{array}$

validation models. This gives some insights about the absence of randomness of the model, thereby assuring reliability and stability. When changing some molecules from the active group to the inactive one, the predictive capability decreases. Hence, it can be concluded that the presence of a particular molecule does not alter the $\mathrm{DF}_{2}$ value significantly. Moreover, in the validation models, both the sensitivity and specificity decrease significantly.

Figure $5 \mathrm{~B}$ shows the ROC curve for $\mathrm{DF}_{2}$. The value of AUC $=0.946$ represents a probability of $94.6 \%$ of correct classification with $\mathrm{DF}_{2}$.

Next, Figure 6B shows the ADD for $\mathrm{DF}_{2}$. Here, the domain of applicability ranges between -6 and 6 . In addition, information about the range of $\mathrm{DF}_{2}$ in which a higher expectancy of finding inhibitors of the formation of chitin $(1.5-6)$ is depicted. Moreover, the overlap zone ( -2.5 and $1.5)$ between the active and inactive compounds is present. $\mathrm{DF}_{2}$ values $<-6$ and $>6$ will be considered outside the range of applicability of the model and are therefore considered as nonclassifiable by the model.

Virtual Screening. After validating the predictive models and identifying the range of application, the commercial database, The Natural Product Collection from MicroSource Discovery System Inc., ${ }^{17}$ was screened, searching for new fungicides and chitin formation inhibitors of natural origin.

Table S3 in the Supporting Information shows the $\mathrm{DF}_{1} y$ $\mathrm{DF}_{2}$ values obtained for each natural compound of the dataset (some 700 molecules).

In Tables 3 and S4 is reported the selection using the two discriminant equations $\mathrm{DF}_{1}$ and $\mathrm{DF}_{2}$, respectively. A molecule will be considered active if the following conditions of $\mathrm{ADD}$ are met: $\mathrm{DF}_{1}$ values from 0.5 to 4 and $\mathrm{DF}_{2}$ from 1.5 to 6 .

Only 16 compounds pass the filter of the topological model for the selection of potential natural fungicides by the inhibition of chitin formation ( $2.3 \%$ of the screen data). In addition, 13 of the 16 selected compounds (81.2\%) have already been described with antifungal activity in the literature, suggesting that the topological model presented is highly predictive. 
Table 3. Selection of Potential Natural Fungicides by Inhibiting Chitin Formation

\begin{tabular}{|c|c|c|c|c|}
\hline compound & Cas\# no & $\mathrm{DF}_{1}$ & $\mathrm{DF}_{2}$ & reported activity \\
\hline 1-methylxanthine & $6136-37-4$ & 2.14 & 1.47 & no info registered ${ }^{a}$ \\
\hline 4-O-methylphloracetophenone & $7507-89-3$ & 3.19 & 2.80 & 30 \\
\hline antheraxanthin & $640-03-9$ & 2.87 & 3.01 & no info registered \\
\hline atranorin & $479-20-9$ & 0.52 & 2.38 & 31 \\
\hline baeomycesic acid & 644-66-6 & 0.69 & 2.07 & 32 \\
\hline camphor & $76-22-2$ & 3.27 & 4.73 & 33 \\
\hline cantharidin & $56-25-7$ & 0.64 & 6.76 & 34 \\
\hline evernic acid & $537-09-7$ & 1.07 & 3.55 & 35 \\
\hline everninic acid & $570-10-5$ & 3.19 & 2.74 & 36 \\
\hline hematommic acid & $479-25-4$ & 0.56 & 1.93 & no info registered \\
\hline lecanoric acid & $480-56-8$ & 0.62 & 4.09 & 37 \\
\hline menthol $(-)$ & $1490-04-6$ & 1.46 & 4.60 & 38 \\
\hline Menthone & $14073-97-3$ & 1.51 & 4.60 & 39 \\
\hline methyl orsellinate & $3187-58-4$ & 3.08 & 2.11 & 40 \\
\hline orsellinic acid & $480-64-8$ & 3.29 & 3.96 & 41 \\
\hline orsellinic acid, ethyl ester & $2524-37-0$ & 0.73 & 2.34 & 42 \\
\hline
\end{tabular}

${ }^{a}$ As far as we know, no information about antifungal or chitin inhibition activity is present in the literature.

Figure 8 shows the chemical structure of three new potential fungicides acting as the inhibitors of chitin formation, identified using $\mathrm{DF}_{1}$ and $\mathrm{DF}_{2}$.

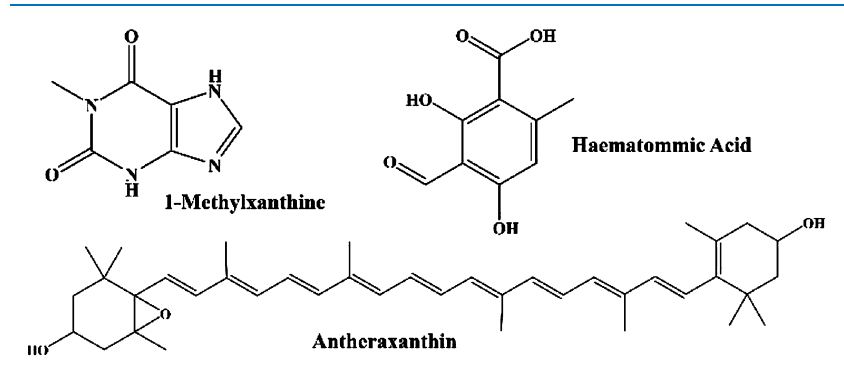

Figure 8. Potential fungicides and chitin formation inhibitors of natural origin.

Even if the antifungal activity for the three molecules is not recorded in the literature, these natural compounds had been reported to show some pharmacological activity. 1-Methylxanthine, well known as the major metabolite of caffeine, has positive effects against asthma and diuretic activity. ${ }^{43}$ Hematommic acid is described as an effective antioxidant agent, ${ }^{44}$ as well as antheraxanthin, which also has a carotenoid profile. $^{45}$

A similarity study on the three potential fungicides is carried out using SciFinder Scholar and ChemSpider database. Tanimoto and Euclidean parameters ${ }^{46}$ are used, searching for similarity in a range between 80 and $99 \%$ among the commercially available molecules. For 1-methylxanthine, no antifungal activity among similar molecules was found; however, several methyl xanthine derivatives are described in the literature as fungicides, with activity as chitin inhibitors. $^{47,48}$ As for the antheraxanthin similar, no antifungal activity is described, while for the haematommic acid one molecule, the 2,4-dihydroxy-6-methylbenzoic acid (CAS number: $480-64-8$ ) with a similarity of $82 \%$, is reported as antibacterial and antifungal. ${ }^{49,50}$

\section{CONCLUSIONS}

In the present work, the authors show how MT can be a key paradigm in developing QSAR strategies, searching for new fungicides. Considering the urgent situation in crop field protection, they decided to develop an in silico strategy to search new molecules capable to inhibit the synthesis of chitin in fungi. To do so, the authors calculated two discriminant equations using statistical LDA. The two equations were validated with external and internal methods and then used for the screening of The Natural Product Collection from MicroSource Discovery, ${ }^{17}$ searching for the potential inhibitors of the formation of chitin. Only 16 molecules pass the topological filters, and almost $80 \%$ of the candidates was later confirmed, as already described in the literature, for some antifungal activity. Three best candidates are selected as new, natural agents to control fungi growth in crop protection: 1methylxanthine, hematommic acid, and antheraxanthin. In the authors' opinion, the present results offer an insight about the potential of MT as an effective methodology in designing new agrochemical agents. The identification of the three new potential fungicides that inhibit the chitin synthesis may be an attractive starting point for the development of new products in crop protection, above all, considering that the molecules are natural products.

\section{ASSOCIATED CONTENT}

\section{Supporting Information}

The Supporting Information is available free of charge at https://pubs.acs.org/doi/10.1021/acsomega.0c00177.

All indices calculated with Dragon software version 5.4. ${ }^{15}$ and their values for the selected equations for every compound (training set, external test set, and virtual screening set) (XLSX)

\section{AUTHOR INFORMATION}

\section{Corresponding Author}

Ramon Garcia-Domenech - Molecular Topology and Drug Design Unit, Department of Physical Chemistry, University of Valencia, Valencia 46010, Spain; 이이이.org/0000-00031137-0587; Phone: +34-963544291; Email: ramon.garcia@ uv.es

\section{Authors}

Maria Galvez-Llompart - Instituto de Tecnologia Quimica, UPV-CSIC, Universidad Politecnica de Valencia, Valencia E- 
46022, Spain; Molecular Topology and Drug Design Unit, Department of Physical Chemistry, University of Valencia, Valencia 46010, Spain

Riccardo Zanni - Departamento de Microbiologia, Facultad de Ciencias, Universidad de Malaga, Malaga 29071, Spain; Molecular Topology and Drug Design Unit, Department of Physical Chemistry, University of Valencia, Valencia 46010, Spain

Jorge Galvez - Molecular Topology and Drug Design Unit, Department of Physical Chemistry, University of Valencia, Valencia 46010, Spain

Complete contact information is available at: https://pubs.acs.org/10.1021/acsomega.0c00177

\section{Author Contributions}

The manuscript was written through contributions of all authors.

\section{Notes}

The authors declare no competing financial interest.

\section{ACKNOWLEDGMENTS}

The authors acknowledge the MINECO (Spanish Ministry of Economy, Industry and Competitivity) Project: "Desarrollo de nuevas herramientas para el control de oidios" (AGL201676216-C2-2-R). Maria Galvez also acknowledges the postdoctoral grant from Generalitat Valenciana and Fondo Social Europeo reference: APOST/2019/055.

\section{ABBREVIATIONS}

MOA, mechanism of action; QSAR, quantitative structureactivity relationship; MT, molecular topology; LDA, linear discriminant analysis; $\mathrm{DF}$, discriminant function; $\mathrm{ADD}$, activity distribution diagram; ROC, receiver operating characteristic; AUC, area under the curve

\section{REFERENCES}

(1) Almeida, F.; Rodrigues, M. L.; Coelho, C. The Still Underestimated Problem of Fungal Diseases Worldwide. Front. Microbiol. 2019, 10, 214.

(2) Avery, S. V.; Singleton, I.; Magan, N.; Goldman, G. H. The fungal threat to global food security. Fungal Biol. 2019, 123, 555-557.

(3) Chaudhary, P. M.; Tupe, S. G.; Deshpande, M. V. Chitin Synthase Inhibitors as Antifungal Agents. Mini-Rev. Med. Chem. 2013, 13, 222-236.

(4) Cohen, E. Chitin Synthesis and Degradation as Targets for Pesticide Action. Arch. Insect Biochem. Physiol. 1993, 22, 245-261.

(5) Hartl, L.; Zach, S.; Seidl-Seiboth, V. Fungal chitinases: diversity, mechanistic properties and biotechnological potential. Appl. Microbiol. Biotechnol. 2012, 93, 533-543.

(6) Batran, R. Z.; Khedr, M. A.; Abdel Latif, N. A.; Abd El Aty, A. A.; Shehata, A. N. Synthesis, homology modeling, molecular docking, dynamics, and antifungal screening of new 4-hydroxycoumarin derivatives as potential chitinase inhibitors. J. Mol. Struct. 2019, $1180,260-271$.

(7) Marx, J. L. Chitin Synthesis Inhibitors: New Class of Insecticides. Science 1977, 197, 1170-1172.

(8) Galvez, J.; Galvez-Llompart, M.; Garcia-Domenech, R. Introduction to Molecular Topology: Basic Concepts and Application to Drug Design. Curr. Comput.-Aided Drug Des. 2012, 8, 196-223.

(9) García-Domenech, R.; Gálvez, J.; de Julián-Ortiz, J. V.; Pogliani, L. Some New Trends in Chemical Graph Theory. Chem. Rev. 2008, 108, 1127-1169.

(10) de Julián-Ortiz, J. V.; de Gregorio Alapont, C.; RíosSantamarina, I.; García-Doménech, R.; Gálvez, J. Prediction of properties of chiral compounds by molecular topology. J. Mol. Graphics Modell. 1998, 16, 14-18.

(11) Zanni, R.; Galvez-Llompart, M.; Machuca, J.; GarciaDomenech, R.; Recacha, E.; Pascual, A.; Rodriguez-Martinez, J. M.; Galvez, J. Molecular topology: A new strategy for antimicrobial resistance control. Eur. J. Med. Chem. 2017, 137, 233-246.

(12) Pérez-García, A.; Martínez-Cruz, J.; Zanni, R.; RomeroHinojosa, D.; Fernández-Ortuño, D.; Galvez-Llompart, M.; GarciaDomenech, R.; Galvez, J. Chitin deacetylase inhibitors and their use as agricultural fungicides, arthropocides and nematicides. P 201930440, 2019, Spain.

(13) Blay, V.; Gullón-Soleto, J.; Gálvez-Llompart, M.; Gálvez, J.; García-Domenech, R. Biodegradability Prediction of Fragrant Molecules by Molecular Topology. ACS Sustainable Chem. Eng. 2016, 4, 4224-4231.

(14) Council, BCP. A World Compendium: The Pesticide Manual, 6th ed.; British Crop Protection Council: Hampshire, U.K., 2012.

(15) Dragon for Windows (Software for Molecular Descriptor Calculations), version 5.4.; Talete srl.: Milan, Italy, 2006.

(16) StatSoft. Statistica (Data Analysis Software System), version 9; Tulsa, USA, 2009.

(17) The Natural Product Collection from MicroSource Discovery System Inc. http://www.msdiscovery.com/natprod.html (accessed 2020-04-15).

(18) Nitoda, T.; Usuki, H.; Kurata, A.; Kanzaki, H. Macromolecular Insect Chitinase Inhibitors Produced by Fungi: Screening and Partial Characterization. J. Pestic. Sci. 2003, 28, 33-36.

(19) Hirose, T.; Sunazuka, T.; Sugawara, A.; Endo, A.; Iguchi, K.; Yamamoto, T.; Ui, H.; Shiomi, K.; Watanabe, T.; Sharpless, K. B.; Ōmura, S. Chitinase inhibitors: extraction of the active framework from natural argifin and use of in situ click chemistry. J. Antibiot. 2009, 62, 277-282.

(20) Zanni, R.; Galvez-Llompart, M.; García-Domenech, R.; Galvez, $\mathrm{J}$. Latest advances in molecular topology applications for drug discovery. Expert Opin. Drug Discovery 2015, 10, 945-957.

(21) Cousins, K. R. Computer review of ChemDraw Ultra 12.0. J. Am. Chem. Soc. 2011, 133, 8388.

(22) Todeschini, R.; Consonni, V. Handbook of Molecular Descriptors, 2nd ed.; Methods and Principles in Medicinal Chemistry; Wiley-VCH: Verlag GmbH: Weinheim, 2008; pp 668.

(23) Izenman, A. J. Linear discriminant analysis. Anonymous Modern Multivariate Statistical Techniques. Modern Multivariate Statistical Techniques: Regression, Classification, and Manifold Learning, 1st ed.; Springer Publishing Company, Incorporated, 2013; pp 237280.

(24) De Maesschalck, R.; Jouan-Rimbaud, D.; Massart, D. L. The mahalanobis distance. Chemom. Intell. Lab. Syst. 2000, 50, 1-18.

(25) James, G.; Witten, D.; Hastie, T.; Tibshirani, R. An Introduction to Statistical Learning: with Applications in R; Springer: New York, 2013.

(26) Furnival, G. M. All possible regressions with less computation. Technometrics 1971, 13, 403-408.

(27) Kumar, R.; Indrayan, A. Receiver Operating Characteristic (ROC) Curve For Medical Researchers. Indian J. Pediatr. 2011, 48, 277-287.

(28) Gálvez, J.; García-Domenech, R.; de Gregorio Alapont, C.; de Julián-Ortiz, J. V.; Popa, L. Pharmacological distribution diagrams: a tool for de novo drug design. J. Mol. Graphics 1996, 14, 272-276.

(29) Lipinski, P. F. J.; Szurmak, P. SCRAMBLE'N'GAMBLE.: a tool for fast and facile generation of random data for statistical evaluation of QSAR models. Chem. Zvesti 2017, 71, 2217-2232.

(30) Anetai, M.; Hasegawa, S.; Kaneshima, H. Antifungal constituent from leaves of Rhododendron dauricum L. Nat. Med. 1995, 49, 217.

(31) Goel, M.; Dureja, P.; Rani, A.; Uniyal, P. L.; Laatsch, H. Isolation, Characterization and Antifungal Activity of Major Constituents of the Himalayan Lichen Parmelia reticulata Tayl. J. Agric. Food Chem. 2011, 59, 2299-2307. 
(32) Wang, X.-n.; Yu, W.-t.; Lou, H.-x. Antifungal Constituents from the Chinese MossHomalia trichomanoides. Chem. Biodiversity 2005, 2, 139-145.

(33) Mahilrajan, M.; Jeyarani, N.; Robika, K.; Nilushiny, A. M.; SriThayalan, S. Screening the antifungal activity of essential oils against decay fungi from palmyrah leaf handicrafts. Biol. Res. 2014, 47, 35.

(34) Deshpande, M. V.; O’Donnell, R.; Gooday, G. W. Regulation of chitin synthase activity in the dimorphic fungus Benjaminiella poitrasii by external osmotic pressure. FEMS Microbiol. Lett. 2006, 152, 327332.

(35) Halama, P.; Van Haluwin, C. Antifungal activity of lichen extracts and lichenic acids. BioControl 2004, 49, 95-107.

(36) Fujikawa, F.; Hitosa, Y.; Yagi, Y.; Nakazawa, S.; Omatsu, T. Antibacterial and antifungal action of lichen substances and their derivatives. Yakugaku Zasshi 1957, 77, 307-309.

(37) Thadhani, V. M.; Choudhary, M. I.; Khan, S.; Karunaratne, V. Antimicrobial and toxicological activities of some depsides and depsidones. J. Natl. Sci. Found. 2012, 40, 43-48.

(38) Singh, M.; Kumar, S.; Kumar, A.; Kumar, P.; Narasimhan, B. Synthesis, antimicrobial evaluation, and QSAR analysis of 2-isopropyl5-methylcyclohexanol derivatives. Med. Chem. Res. 2012, 21, 511522.

(39) Makkar, M. K.; Sharma, S.; Kaur, H. Evaluation of Mentha arvensis essential oil and its major constituents for fungitoxicity. $J$. Food Sci. Technol. 2018, 55, 3840-3844.

(40) Liu, B.-L.; Hu, X.; He, H.-L.; Qiu, L.; Li, Y.-Z.; Ding, W.-B. A new epicatechin glucopyranoside derivative from Styrax suberifolius. Nat. Prod. Res. 2019, 7, 1-7.

(41) Malquichagua Salazar, K. J.; Delgado Paredes, G. E.; Lluncor, L. R.; Young, M. C. M.; Kato, M. J. Chromenes of polyketide origin from Peperomia villipetiola. Phytochemistry 2005, 66, 573-579.

(42) Bohnert, M.; Nützmann, H.-W.; Schroeckh, V.; Horn, F.; Dahse, H.-M.; Brakhage, A. A.; Hoffmeister, D. Cytotoxic and antifungal activities of melleolide antibiotics follow dissimilar structure-activity relationships. Phytochemistry 2014, 105, 101-108.

(43) González-Domínguez, R.; Urpi-Sarda, M.; Jáuregui, O.; Needs, P. W.; Kroon, P. A.; Andrés-Lacueva, C. Quantitative Dietary Fingerprinting (QDF)-A Novel Tool for Comprehensive Dietary Assessment Based on Urinary Nutrimetabolomics. J. Agric. Food Chem. 2020, 68, 1851.

(44) Whang, W. K.; Park, H. S.; Ham, I.; Oh, M.; Namkoong, H.; Kim, H. K.; Hwang, D. W.; Hur, S. Y.; Kim, T. E.; Park, Y. G.; Kim, J.R.; Kim, J. W. Methyl gallate and chemicals structurally related to methyl gallate protect human umbilical vein endothelial cells from oxidative stress. Exp. Mol. Med. 2005, 37, 343-352.

(45) Lux, P. E.; Carle, R.; Zacarías, L.; Rodrigo, M.-J.; Schweiggert, R. M.; Steingass, C. B. Genuine Carotenoid Profiles in Sweet Orange [Citrus sinensis (L.) Osbeck cv. Navel] Peel and Pulp at Different Maturity Stages. J. Agric. Food Chem. 2019, 67, 13164.

(46) Bajusz, D.; Rácz, A.; Héberger, K. Why is Tanimoto index an appropriate choice for fingerprint-based similarity calculations? J. Cheminf. 2015, 7, 20.

(47) Rao, F. V.; Andersen, O. A.; Vora, K. A.; Demartino, J. A.; van Aalten, D. M. F. Methylxanthine drugs are chitinase inhibitors: investigation of inhibition and binding modes. Chem. Biol. 2005, 12, 973-980.

(48) Tsirilakis, K.; Kim, C.; Vicencio, A. G.; Andrade, C.; Casadevall, A.; Goldman, D. L. Methylxanthine inhibit fungal chitinases and exhibit antifungal activity. Mycopathologia 2012, 173, 83-91.

(49) Amborabé, B.-E.; Fleurat-Lessard, P.; Chollet, J.-F.; Roblin, G. Antifungal effects of salicylic acid and other benzoic acid derivatives towards Eutypa lata: Structure-activity relationship. Plant Physiol. Biochem. 2002, 40, 1051-1060.

(50) O Hanuš, L.; Temina, M.; Dembitsky, V. Antibacterial and antifungal activities of some phenolic metabolites isolated from the lichenized ascomycete Ramalina lacera. Nat. Prod. Commun. 2008, 16, 677-688. 